${ }^{1}$ Departamento de Nefrología, Escuela de Medicina, Facultad de Medicina Pontificia Universidad Católica de Chile. Santiago, Chile. 2Departamento de Nefrología, Hospital de Talca, Facultad de Medicina Universidad Católica del Maule. Talca, Chile.

Trabajo no recibió financiamiento. Los autores declaran no tener conflictos de interés.

Recibido el 11 de enero de 2019, aceptado el 17 de septiembre de 2019.

Correspondencia a: Dr. Rodrigo A. Sepúlveda Departamento de Nefrología. Diagonal Paraguay 362, piso 4. Santiago, Chile. rrsepulveda@uc.cl

\section{Intoxicación por etilenglicol, fisiopatología y enfrentamiento clínico}

\author{
RODRIGO A. SEPÚLVEDA ${ }^{1}$, ESPERANZA SELAMÉ2, \\ ERIC ROESSLER ${ }^{1}$, RODRIGO TAGLE $^{1}$, ANDRÉS VALDIVIESO ${ }^{1}$
}

\section{Ethylene glycol poisoning. An update}

Toxic alcohols can produce severe poisoning with multiple organic involvement and even death. The most common form is ethylene glycol. The diagnosis can be extremely difficult if there is no history of its consumption. Its clinical presentation can simulate other conditions. Ethylene glycol poisoning is characterized by an initial rise in plasma osmolal gap that decreases during the evolution, while alcohol is metabolized to acids. This last condition causes a metabolic acidosis with elevated anion gap. The clinical manifestations are diffuse neurological involvement initially, followed by hemodynamic alterations due to myocardial damage associated with hypocalcemia and acidemia. Subsequently, severe tubular renal damage appears, which may require renal replacement therapy, and finally, focal neurological alterations. To treat this poisoning, it is necessary to inhibit the transformation of alcohol into acids, increase the metabolism of the latter or withdraw them directly with hemodialysis.

(Rev Med Chile 2019; 147: 1572-1578)

Key words: Alcohols; Ethylene Glycol; Poisoning; Therapeutics.

\section{H} istóricamente, los alcoholes han sido de gran utilidad para el ser humano. Cuando se habla de alcoholes, la mayoría de las personas piensa en etanol, cuyo destino se ha desvirtuado gracias al consumo irresponsable, muchas veces perjudicial para la salud y con impacto negativo hacia la sociedad. Sin embargo, los alcoholes son mucho más que una sustancia "beneficiosa" para las conductas festivas de la sociedad humana. Y es que sus fantásticas propiedades físico-químicas han permitido el desarrollo tecnológico e industrial de la humanidad. Los alcoholes poseen un muy bajo punto de congelación por lo que son ampliamente utilizados en la industria automotriz (anticongelantes, líquido de freno, combustibles). Por otro lado, al encontrarse en estado líquido a temperatura ambiental, permiten su utilización como solventes. El agua es una molécula polar que admite sólo la disolución en ella de sustancias polares. En cambio, los alcoholes poseen un grupo polar hidroxilo $(-\mathrm{OH})$ y una estructura hidrocarbonada apolar, así pueden interactuar tanto con moléculas polares como apolares. Esta propiedad permite que los alcoholes se empleen como solvente de pigmentos, pinturas, odoríferos, detergentes, fármacos, cosméticos, entre otros químicos. Además, pueden alterar estructuras celulares vegetativas actuando como antimicrobianos (desinfectantes, sanitizadores, dentríficos).

El problema radica en que algunos de estos alcoholes, cuando son incorporados al organismo, producen múltiples trastornos que pueden ir desde daños orgánicos reversibles hasta la muerte de una persona ${ }^{1}$. Definimos a estos alcoholes como "alcoholes tóxicos".

La amplia disponibilidad de alcoholes tóxicos en el mercado favorece cuadros graves de intoxicación; ya sea por ingestión, inhalación o contacto con la piel. Lo anterior puede ocurrir como accidente (principalmente en población pediátrica) o con fines suicidas ${ }^{2}$.

Los principales alcoholes tóxicos son el metanol, etilenglicol, dietilenglicol, propilenglicol 
e isopropanol. La incidencia en Estados Unidos de envenenamiento por cualquier sustancia es de 17,6 por 1.000 habitantes; de éstos, 5,3\% se debe a un alcohol tóxico ${ }^{3}$.

En el organismo humano, los alcoholes actúan como una molécula osmóticamente activa generando hiperosmolalidad y elevación del gap osmolal plasmático (GOP). Algunos alcoholes inducen daño en forma directa (propilenglicol, isopropanol y etanol). Pero también, el efecto dañino puede ser indirecto, ya que los alcoholes son metabolizados en el hígado a un ácido capaz de alterar el funcionamiento celular. Las intoxicaciones más graves se deben a metanol, etilenglicol y dietilenglicol. Todos ellos elevan el GOP y sus metabolitos producirán daño orgánico más una acidosis metabólica con anion gap (AG) elevado. $\mathrm{El}$ isopropanol raramente produce cuadros de acidosis (cetoacidosis), siendo el mecanismo de daño directamente generado por el alcohol. El propilenglicol se transforma en ácido láctico, la intoxicación es iatrogénica y raramente se asocia con cuadros graves ${ }^{1}$.

En esta revisión, expondremos las propiedades físico-químicas del etilenglicol, su comportamiento en el organismo humano y los mecanismos por los cuales induce daño. Además, se explicitará el cuadro clínico, las medidas diagnósticas y terapéuticas para evitar las graves repercusiones asociadas a la intoxicación por etilenglicol.

\section{Etilenglicol}

El etilenglicol o etanodiol posee 2 grupos hidroxilo y tiene un peso molecular de $62,1 \mathrm{~g} / \mathrm{mol}$. Puede disolverse en agua y como solvente es un líquido transparente, espeso, no volátil, incoloro, inodoro y con sabor dulce ${ }^{1}$. Por lo anterior, es necesario añadirle colorantes para reconocerlo visualmente y evitar intoxicaciones (rojo fenol o fluoresceína).

Se utiliza como anticongelante en equipos de refrigeración, en motores de combustión interna, líquido de frenos, solvente para transferencia de calor, sistemas de protección antihielo, revelado de fotografías y como solvente de plásticos y pigmentos.

Es el alcohol tóxico más frecuentemente implicado en intoxicaciones: $2 \%$ de todos los envenenamientos con una mortalidad cercana al $20 \%{ }^{4-6}$.

\section{Farmacocinética}

La mayoría de las intoxicaciones ocurre por ingestión directa de etilenglicol. A diferencia de otros alcoholes, no atraviesa la piel sana; sólo se puede absorber por vía cutánea cuando la piel está dañada ${ }^{1}$.

Se absorbe rápidamente desde el tracto gastrointestinal, teniendo una biodisponibilidad del $100 \%$ y un volumen de distribución similar al agua corporal $(0,5$ a $0,8 \mathrm{~L} / \mathrm{kg})$. Las concentraciones plasmáticas máximas ocurren 30 a $60 \mathrm{~min}$ posteriores a la ingestión ${ }^{7}$. Se estima que por cada $10 \mathrm{mg} / \mathrm{dL}$ de concentración sérica de etilenglicol, la osmolalidad plasmática aumenta en $1,8 \mathrm{mOsm} / \mathrm{kg}^{1}$.

La eliminación del etilenglicol comienza inmediatamente, principalmente por metabolización hepática o excreción renal directa.

La oxidación hepática de alcoholes se realiza mediante la enzima alcohol deshidrogenasa $(\mathrm{ADH})$, que transforma el etilenglicol en glicoaldehído. Luego, la enzima aldehído deshidrogenasa cataboliza este último en ácido glicólico. Ambas reacciones enzimáticas reducen el $\mathrm{NAD}^{+}$a $\mathrm{NADH}$, incrementando la relación $\mathrm{NADH} / \mathrm{NAD}^{+}$y promoviendo un estado reducción-oxidación oxidativo en el hepatocito. Luego, el ácido glicólico puede transformarse en ácido glioxílico y ácido oxálico (Figura 1$)^{8}$.

De todo el etilenglicol ingerido, un 20\% será excretado directamente por el riñón, mientras el resto será biotransformado en el hígado, para luego eliminar estos metabolitos en forma de ácidos y/o sales por la orina ${ }^{7}$.

La vida media del etilenglicol son 3 a $8,6 \mathrm{~h}$ con función hepática y renal normal, sin embargo, se puede prolongar a $18 \mathrm{~h}$ si existe consumo de etanol ${ }^{1}$. Esto último se debe a que el etanol tiene una afinidad 10 a 20 veces mayor por la enzima alcohol deshidrogenasa limitando el metabolismo hepático de etilenglicol y disminuyendo la producción de ácidos 9 .

En la Tabla 1 se describen las propiedades químicas y farmacocinéticas del etilenglicol ${ }^{1,7,8,10-12}$.

\section{Efectos sistémicos de la intoxicación}

Se ha reportado que una dosis de etilenglicol de 1,4 a $1,5 \mathrm{~mL} / \mathrm{kg}$ es letal, aunque también puede ser mortal en dosis menores $(30 \mathrm{~mL})^{7}$. 


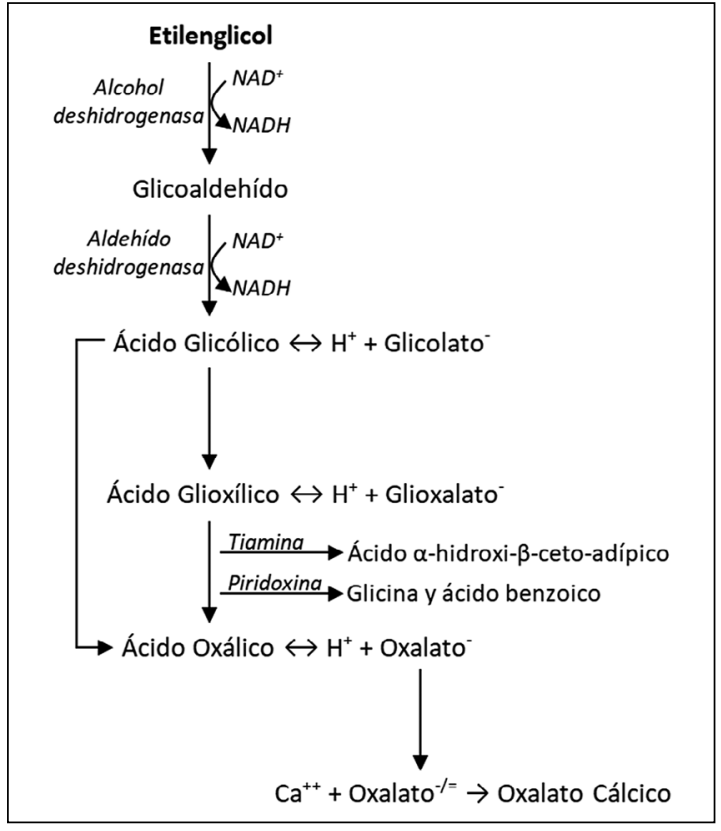

Figura 1. Metabolización hepática de etilenglicol.

Los pacientes con mayor mortalidad en una intoxicación por etilenglicol son aquellos que presentan acidosis metabólica severa $(\mathrm{pH}<7,1)$, inician tratamiento en forma tardía luego de la exposición $(>10 \mathrm{~h})$ y/o se presentan con falla renal ${ }^{1,6}$.

Luego de la ingestión de etilenglicol, su metabolización generará los ácidos (glicólico, glioxílico y oxálico). Todos estos inducirán una acidosis metabólica con AG aumentado.

El glicolato puede afectar la respiración celular induciendo una acidosis láctica tipo $\mathrm{B}^{8}$. Las células tubulares renales son particularmente vulnerables al daño mediado por glicolato, desarrollando necrosis tubular aguda tóxica.

El oxalato precipita con calcio produciendo hipocalcemia y depósitos tisulares en el riñón, corazón, cerebro y pulmón. Clínicamente, esto último se expresa como falla renal aguda, disfunción miocárdica y alteraciones neurológicas ${ }^{2,7}$.

La acidosis láctica puede ser frecuente en estos pacientes ya que la acidemia y los cristales de oxalato de calcio afectan el funcionamiento cardiovascular generando hipoxia tisular (acidosis láctica tipo A). A lo anterior, se agrega el efecto celular del glicolato.

Característicamente los pacientes padecen
Tabla 1. Propiedades químicas y farmacocinéticas del etilenglicol

\begin{tabular}{|ll|}
\hline Peso molecular & $62,1 \mathrm{~g} / \mathrm{mol}$ \\
\hline Vías de ingreso & $\begin{array}{l}\text { Oral } \\
\text { Piel lesionada }\end{array}$ \\
\hline Volumen de distribución & $100 \%$ \\
\hline Concentración máxima & $0,5-0,8 \mathrm{~L} / \mathrm{kg}$ \\
\hline Vida media & $30-60 \mathrm{~m}$ de ingestión \\
& $3-8,6 \mathrm{~h}$ \\
& $17-18 \mathrm{~h}$ con etanol \\
& $20 \mathrm{~h}$ con fomepizol \\
& $2,5-3,5 \mathrm{~h}$ con hemo- \\
diálisis & $1,8 \mathrm{mOsm} / \mathrm{kg}$ \\
Osmolalidad por cada $10 \mathrm{mg} / \mathrm{dL}$ & $80 \%$ hepática \\
Eliminación & $20 \%$ renal \\
& $\begin{array}{l}\text { Ácido glicólico, ácido } \\
\text { glioxílico, ácido oxálico }\end{array}$ \\
Metabolitos & $30 \mathrm{~mL}$ a 1,5 mL/kg \\
\hline Dosis letal &
\end{tabular}

una acidosis metabólica con AG aumentado al momento de presentación, reflejando la adición de bases conjugadas: glicolato, glioxalato y oxalato. A ellas se agregan el lactato (si existe acidosis láctica asociada), sulfatos y fosfatos (cuando hay una falla renal aguda concomitante).

\section{Cuadro clínico}

El cuadro clínico se caracteriza por 3 a 4 etapas $^{13-15}$ :

1. Primeras $12 \mathrm{~h}$ : compromiso neurológico por el efecto osmótico del etilenglicol que induce edema cerebral ${ }^{1,2,14}$. Si bien, los alcoholes provocan hiperosmolalidad en el extracelular, se presume que el etilenglicol podría ingresar al parénquima cerebral ocasionando los trastornos. Habrá euforia, somnolencia, confusión, disartria, ataxia, nistagmus, convulsiones, pudiendo llegar al coma y muerte ${ }^{1,2}$. Se han descrito signos neurológicos focales luego de las $12 \mathrm{~h}$ (posiblemente por el depósito de cristales de oxalato de calcio $)^{1}$. Inicialmente, puede haber náuseas y vómitos por irritación gástrica $^{14}$. 
2. Segunda etapa (12-36 h postingestión): los depósitos de oxalato cálcico, más la hipocalcemia generan depresión miocárdica que se agrava con la acidemia. Puede haber hiperreflexia, espasmos musculares y prolongación del QT por hipocalcemia ${ }^{14}$.

3. Tercera etapa (2-3 días después): falla renal, por daño tubular directo asociado a glicolato y precipitación de cristales de oxalato cálcico intratubulares. Hay dolor lumbar, proteinuria, hematuria, oliguria e incluso anuria. La falla renal aunque puede ser grave y requerir hemodiálisis, generalmente se recupera en algunas semanas ${ }^{14}$. Cuando el paciente se presenta tardíamente y no es posible obtener el antecedente de consumo de etilenglicol, una falla renal aguda aislada puede ser la única forma de presentación; por este motivo se recomienda sospechar y buscar intoxicación por etilenglicol en toda falla renal aguda sin etiología, sobre todo, si se acompaña de síntomas neurológi$\cos ^{15}$.

4. Tardíamente (después de 1 semana) puede haber una última etapa que no es constante y se caracteriza por neuropatía que afecta pares craneanos $^{16,17}$. Generalmente es transitoria y se debe al depósito de cristales de oxalato cálcico.

\section{Diagnóstico}

El diagnóstico de una intoxicación por alcoholes puede ser muy complejo, sobre todo, cuando la presentación es tardía y el paciente no aporta el antecedente. Inicialmente, existe un gran GOP y acidosis metabólica con AG aumentado (presente en $86 \%$ de los casos) $)^{4}$. Conforme el cuadro progresa y se metaboliza el alcohol, disminuye el GOP y empeora la acidosis ${ }^{18}$. Cuando se agrega falla renal esta evolución "clásica" se pierde, ya que se acumulan ácidos fijos (aumento del AG) y productos nitrogenados (incremento del GOP). Se estima que el GOP en sujetos normales es 8-9 $\mathrm{mOsm} / \mathrm{kg}$, en falla renal aguda $12 \mathrm{mOsm} / \mathrm{kg}$ y $20 \mathrm{mOsm} / \mathrm{kg}$ cuando la falla renal es crónica ${ }^{19}$. Múltiples fármacos pueden elevar levemente el GOP (glicerol, glicina, maltosa, manitol, radiocontrastes, sorbitol) ${ }^{20,21}$. Un GOP > $10 \mathrm{mOsm} /$ $\mathrm{kg}$ es sospechoso de intoxicación por alcoholes ${ }^{22}$, mientras un valor $>20 \mathrm{mOsm} / \mathrm{kg}$ es altamente sugerente $^{1}$.
El diagnóstico de intoxicación por etilenglicol se confirma mediante la medición de niveles por cromatografía de gases. Lamentablemente esta técnica es poco asequible y puede tardar días ${ }^{1}$.

Los preparados de etilenglicol, generalmente, contienen fluoresceína para reconocer el solvente y evitar su ingestión ${ }^{23}$. La evaluación de derrames en ropa, piel o análisis de orina con lámpara de Wood puede detectar la fluoresceína mediante luz ultravioleta ${ }^{1,23}$. Posee falsos positivos, sólo es demostrable las primeras horas de una intoxicación y una orina ácida negativiza los resultados. Por lo tanto, su ausencia no descarta la intoxicación ${ }^{1}$.

Los cristales de oxalato cálcico urinarios se encuentran en $45 \%$ de los pacientes ${ }^{24}$. Aparecen desde las 4-8 h de la ingestión y persisten por $40 \mathrm{~h}$ en ausencia de falla renal y hasta 4 días en su presencia ${ }^{1}$.

El lactato puede elevarse por hipoperfusión sistémica, alteración de la respiración celular mediada por glicolato y el estado celular oxidativo (incremento relación $\mathrm{NADH} / \mathrm{NAD}^{+}$). Los laboratorios, generalmente, miden lactato mediante reacción con lactato deshidrogenasa; mientras los analizadores de gases inmediatos o al pie de cama, emplean una reacción con lactato oxidasa. Esta última reacción también detecta glicolato (falso positivo). Por este motivo el Gap de lactato podría ser otra herramienta diagnóstica en la intoxicación por etilenglicol ${ }^{25}$.

En cuanto a la gravedad de la intoxicación, los niveles de etilenglicol no permiten predecir la aparición de falla renal o neurotoxicidad. Sin embargo, niveles de glicolato $>10 \mathrm{mmol} / \mathrm{L}$ predicen con un $100 \%$ de sensibilidad el desarrollo de falla renal aguda. Del mismo modo, un AG $>20$ $\mathrm{mmol} / \mathrm{L}$ y/o un $\mathrm{pH}$ plasmático $<7,3$ al momento de presentación, son indicativos de que el paciente desarrollará falla renal aguda ${ }^{6}$. Lo anterior puede ser útil al momento de tomar decisiones terapéuticas.

\section{Tratamiento}

El lavado gástrico, emesis inducida y carbón activado son de escasa utilidad pues deben ser instaurados los primeros $60 \mathrm{~min}$ luego de una ingestión ${ }^{1}$.

La expansión del intravascular aumenta la 
excreción urinaria de etilenglicol mediante inducción de poliuria y, además, previene la precipitación de oxalato cálcico.

La administración de bicarbonato de sodio será necesaria en casos de acidosis metabólica severa, además, inhibe la unión de oxalato y calcio. Sin embargo, puede empeorar la hipocalcemia. Se debe aportar calcio sólo si existen síntomas de hipocalcemia para evitar mayor precipitación sistémica con oxalato.

Como se mencionó anteriormente, el daño en la intoxicación por etilenglicol es producido por los ácidos. Tanto el etanol y fomepizol poseen mayor afinidad por la enzima ADH hepática evitando la generación de ácidos. La afinidad del fomepizol hacia la enzima $\mathrm{ADH}$ es 500 a 1.000 veces superior al etanol y sin toxicidad asociada ${ }^{26}$. Lamentablemente, este fármaco es poco disponible.

Se debe administrar inhibidores de la $\mathrm{ADH}$ (etanol o fomepizol) ante todo paciente con historia de consumo de etilenglicol más un GOP $>10 \mathrm{mOsm} / \mathrm{kg}$. También cuando existe sospecha de consumo de etilenglicol asociado a 2 o más de

\section{Tabla 2. Indicaciones de etanol o fomepizol en intoxicación por etilenglicol}

Niveles de etilenglicol $>20 \mathrm{mg} / \mathrm{dL}$

Historia de consumo de etilenglicol más un GOP $>10$ $\mathrm{mOsm} / \mathrm{kg}$

Sospecha de consumo de etilenglicol más 2 de los siguientes:

- pH arterial < 7,3

- $\mathrm{HCO}_{3}$ - plasmático $<20 \mathrm{mEq} / \mathrm{L}$

- GOP > $10 \mathrm{mOsm} / \mathrm{kg}$

- Cristales de oxalato cálcico urinarios los siguientes: $\mathrm{pH}$ arterial $<7,3 ; \mathrm{HCO}_{3}{ }^{-}$plasmático $<20 \mathrm{mEq} / \mathrm{L}, \mathrm{GOP}>10 \mathrm{mOsm} / \mathrm{kg}$ y/o cristales de oxalato cálcico urinarios (Tabla 2$)^{27}$. Como la medición de niveles plasmáticos de etilenglicol y/o glicolato es poco accesible; es posible utilizar el AG o GOP como monitorización de efectividad en la terapia. Se recomienda mantener el tratamiento con inhibidores de la $\mathrm{ADH}$ hasta que se normalice el pH y el GOP; o los niveles de etilenglicol sean $<10-15 \mathrm{mg} / \mathrm{dL}$. En la Tabla 3 se explicitan las dosis de etanol para tratamiento de intoxicación por etilenglicol; su utilización debiera acompañarse de monitorización seriada con niveles de etanol para lograr una concentración aproximada a 100 $\mathrm{mg} / \mathrm{dL}$, y así, evitar su toxicidad ${ }^{1}$.

La hemodiálisis intermitente es el método más eficiente para extraer etilenglicol y sus metabolitos. La Tabla 4 resume cuándo se recomienda instaurar ${ }^{27}$. Los procedimientos convectivos y continuos son poco eficientes, al igual que la diálisis peritoneal ${ }^{1}$.

La piridoxina intravenosa (IV) en dosis de $50 \mathrm{mg}$ cada $6 \mathrm{~h}$ promueve la metabolización de glioxalato a glicina y ácido benzoico. Del mismo modo, la tiamina IV ( 100 mg cada 6 h), también promueve el metabolismo del glioxalato ${ }^{14,27}$.

Son factores de mal pronóstico un $\mathrm{pH}<7,1$, niveles de etilenglicol $>50$ a $100 \mathrm{mg} / \mathrm{dL}$, niveles de glicolato $>10 \mathrm{mmol} / \mathrm{L}$, falla renal aguda que requiere hemodiálisis y diagnóstico posterior a las $10 \mathrm{~h}$ de ingestión ${ }^{1}$.

\section{Conclusión}

La intoxicación por etilenglicol es poco frecuente, pero potencialmente letal y tratable, por lo que debe ser conocida por médicos que trabajan en unidades de urgencia y de pacientes críticos. Se

Tabla 3. Dosis de etanol en intoxicación por etilenglicol

\begin{tabular}{|lccc|}
\hline Dosis & Etanol 90\% enteral & Etanol 43\% enteral & Etanol 10\% parenteral \\
\hline Carga & $0,86 \mathrm{~mL} / \mathrm{kg}$ & $1,8 \mathrm{~mL} / \mathrm{kg}$ & $7,6 \mathrm{~mL} / \mathrm{kg}$ \\
$\begin{array}{l}\text { Mantención en pacientes } \\
\text { no consumidores de etanol }\end{array}$ & Sin hemodiálisis $0,1 \mathrm{~mL} / \mathrm{kg} / \mathrm{h}$ & Sin hemodiálisis $0,2 \mathrm{~mL} / \mathrm{kg} / \mathrm{h}$ & Sin hemodiálisis $0,83 \mathrm{~mL} / \mathrm{kg} / \mathrm{h}$ \\
Mantención en pacientes & Sin hemodiálisis $0,24 \mathrm{~mL} / \mathrm{kg} / \mathrm{h}$ & Con hemodiálisis $0,5 \mathrm{~mL} / \mathrm{kg} / \mathrm{h}$ & Con hemodiálisis $2,13 \mathrm{~mL} / \mathrm{kg} / \mathrm{h}$ \\
consumidores de etanol & Con hemodiálisis $0,37 \mathrm{~mL} / \mathrm{hg} / \mathrm{h}$ & Sin hemodiálisis $0,46 \mathrm{~mL} / \mathrm{kg} / \mathrm{h}$ & Sin hemodiálisis $1,96 \mathrm{~mL} / \mathrm{kg} / \mathrm{h}$ \\
\hline
\end{tabular}


Tabla 4. Indicaciones de hemodiálisis intermitente en intoxicación por etilenglicol

Indicaciones de Hemodiálisis intermitente en intoxicación por etilenglicol

$\mathrm{pH}<7,25$ que no responde a tratamiento médico

Falla renal severa

Niveles de etilenglicol $>50 \mathrm{mg} / \mathrm{dL}$

debe sospechar cuando existe acidosis metabólica con anion gap aumentado, gap osmolal plasmático elevado, falla renal aguda, cristaluria de oxalato de calcio y/o compromiso neurológico sin explicación evidente.

\section{Referencias}

1. Kraut JA, Kurtz I. Toxic alcohol ingestions: clinical features, diagnosis, and management. Clin J Am Soc Nephrol 2008; 3 (1): 208-25.

2. Leth PM, Gregersen M. Ethylene glycol poisoning. Forensic Sci Int 2005; 155: 179-84.

3. Watson WA, Litovitz TL, Klein-Schwartz W, Rodgers GC Jr, Youniss J, Reid N, et al. 2003 annual report of the American Association of Poison Control Centers Toxic Exposure Surveillance System. Am J Emerg Med 2004; 22 (5): 335-404.

4. Litovitz TL, Klein-Schwartz W, White S, Cobaugh DJ, Youniss J, Omslaer JC, et al. 2000 Annual report of the American Association of Poison Control Centers Toxic Exposure Surveillance System. Am J Emerg Med 2001; 19 (5): 337-95

5. Karlson-Stiber C, Persson H. Ethylene glycol poisoning: experiences from an epidemic in Sweden. J Toxicol Clin Toxicol 1992; 30 (4): 565-74.

6. Porter WH, Rutter PW, Bush BA, Pappas AA, Dunnington JE. Ethylene glycol toxicity: the role of serum glycolic acid in hemodialysis. J Toxicol Clin Toxicol 2001; 39 (6): 607-15.

7. Eder AF, McGrath CM, Dowdy YG, Tomaszewski JE, Rosenberg FM, Wilson RB, et al. Ethylene glycol poisoning: toxicokinetic and analytical factors affecting laboratory diagnosis. Clin Chem 1998; 44 (1): 168-77.

8. Gabow PA, Clay K, Sullivan JB, Lepoff R. Organic acids in ethylene glycol intoxication. Ann Intern Med 1986; 105 (1): $16-20$
9. Chu J, Wang RY, Hill NS. Update in clinical toxicology. Am J Respir Crit Care Med 2002; 166 (1): 9-15.

10. Jacobsen D, Hewlett TP, Webb R, Brown ST, Ordinario AT, McMartin KE. Ethylene glycol intoxication: evaluation of kinetics and crystalluria. Am J Med. 1988; 84 (1): 145-52.

11. Peterson CD, Collins AJ, Himes JM, Bullock ML, Keane WF. Ethylene glycol poisoning: pharmacokinetics during therapy with ethanol and hemodialysis. N Engl J Med 1981; 304 (1): 21-3.

12. Brent J, McMartin K, Phillips S, Burkhart KK, Donovan JW, Wells M, et al. Fomepizole for the treatment of ethylene glycol poisoning. Methylpyrazole for Toxic Alcohols Study Group. N Engl J Med 1999; 340 (11): 832-8.

13. Mokhlesi B, Leikin JB, Murray P, Corbridge TC. Adult toxicology in critical care: Part II: specific poisonings. Chest 2003; 123 (3): 897-922.

14. Brent J. Current management of ethylene glycol poisoning. Drugs 2001; 61 (7): 979-88.

15. Takayesu JK, Bazari H, Linshaw M. Case records of the Massachusetts General Hospital. Case 7-2006. A 47-year-old man with altered mental status and acute renal failure. N Engl J Med 2006; 354 (10): 1065-72.

16. Spillane L, Roberts JR, Meyer AE. Multiple cranial nerve deficits after ethylene glycol poisoning. Ann Emerg Med 1991; 20 (2): 208-10.

17. Reddy NJ, Sudini M, Lewis LD. Delayed neurological sequelae from ethylene glycol, diethylene glycol and methanol poisonings. Clin Toxicol (Phila) 2010; 48 (10): 967-73.

18. Hovda KE, Hunderi OH, Rudberg N, Froyshov S, Jacobsen D. Anion and osmolal gaps in the diagnosis of methanol poisoning: clinical study in 28 patients. Intensive Care Med 2004; 30 (9): 1842-6.

19. Sklar AH, Linas SL. The osmolal gap in renal failure. Ann Intern Med 1983; 98 (4): 481-2.

20. Schelling JR, Howard RL, Winter SD, Linas SL. Increased osmolal gap in alcoholic ketoacidosis and lactic acidosis. Ann Intern Med 1990; 113 (8): 580-2.

21. Mokhlesi B, Leiken JB, Murray P, Corbridge TC. Adult toxicology in critical care: part I: general approach to the intoxicated patient. Chest 2003; 123 (2): 577-92.

22. Smithline N, Gardner KD. Gaps-anionic and osmolal. JAMA 1976; 236 (14): 1594-7.

23. Winter ML, Ellis MD, Snodgrass WR. Urine fluorescence using a Wood's lamp to detect the antifreeze additive sodium fluorescein: a qualitative adjunctive test in suspected ethylene glycol ingestions. Ann Emerg Med 1990; 19 (6): 663-7.

24. Jacobsen D, Akesson I, Shefter E. Urinary calcium oxala- 
te monohydrate crystals in ethylene glycol poisoning. Scand J Clin Lab Invest 1982; 42 (3): 231-4.

25. Meng QH, Adeli K, Zello GA, Porter WH, Krahn J. Elevated lactate in ethylene glycol poisoning: True or false? Clin Chim Acta 2010; 411 (7-8): 601-4.

26. Barceloux DG, Bond GR, Krenzelok EP, Cooper H, Vale JA. American Academy of Clinical Toxicology practice guidelines on the treatment of methanol poisoning. J Toxicol Clin Toxicol 2002; 40 (4): 415-46.

27. Barceloux DG, Krenzelok EP, Olson K, Watson W. American Academy of Clinical Toxicology Practice Guidelines on the Treatment of Ethylene Glycol Poisoning. Ad Hoc Committee. J Toxicol Clin Toxicol 1999; 37 (5): 537-60. 